\title{
MEASUREMENT OF DIFFUSE REFLECTION FACTORS, AND A NEW ABSOLUTE REFLECTOMETER
}

\author{
By A. H. Taylor
}

\section{CONTENTS}

Page

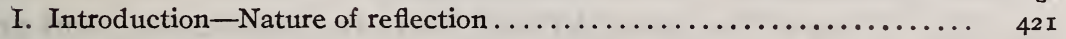

II. Earlier reflectometers $\ldots \ldots \ldots \ldots \ldots \ldots \ldots \ldots \ldots \ldots \ldots \ldots \ldots \ldots \ldots \ldots, 422$

III. The new absolute reflectometer $\ldots \ldots \ldots \ldots \ldots \ldots \ldots \ldots \ldots \ldots \ldots \ldots \ldots .425$

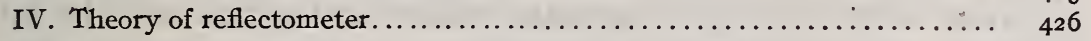

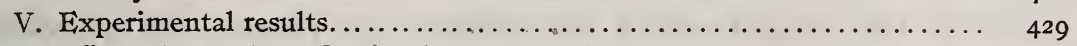

VI. Effect of specular reflection from test surfaces.............. 433

VII. Precautions in use of reflectometer.................... 434

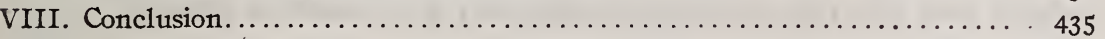

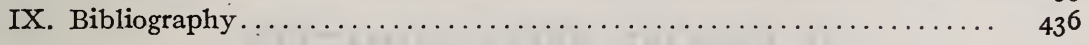

\section{INTRODUCTION-NATURE OF REFLECTION}

The reflection factor of a surface is defined as the ratio of the total luminous flux reflected by the surface to the total luminous flux incident upon it.

The surface may be illuminated by a narrow beam of light, by light from several directions, by totally diffused light, or by some combination of these. Reflection may take place in many wayse. g., specular reflection, in which case an incident cone of light is reflected as a cone, the angles of incidence and reflection being equal; perfectly diffused reflection, in which case the light is reflected in all directions in accordance with the ${ }^{1}$ cosine law of emission; mixed specular and diffused reflection, in all possible combinations between the extremes.

No surface obeys the cosine law of emission perfectly, and most surfaces are very far from being perfect diffusers.

\footnotetext{
1 The cosine law of emission states in substance that the light is reflected from a perfectly diffusing surface in such a manner that the luminous intensity (expressed in candlepower or some similar unit) of an element of area at any angle of emission is equal to the intensity normal to the surface multiplied by the cosine of the angle between the line of emission and the normal to the surface. This assumes that the intensity at any angle is directly proportional to the projected area of the element of surface, hence that the surface brightness (candles per unit area) is constant at all angles.

This law has often been erroneously referred to as Lambert's cosine law. Mr. A. P. Trotter has recently shown (see Bibliography, $x$ ) that Lambert's law refers to the incident light instead of the reflected light, and that his law states that the intensity of illumination of any surface varies as the cosine of the angle between the line of incidence and the normal to the surface. Hence it is seen that Lambert's cosine law is rigidly correct. Mr. Trotter's article also gives much valuable information regarding the characteristics of reflected light.
} 
The numerical value of the reflection factor of a surface may depend on the color of the incident light and the manner of its incidence.

In the practical application of light and illumination it is often desirable to be able to determine the reflection factors of various surfaces. In the design of a lighting installation a knowledge of the reflection factors of the walls and ceilings enables the illuminating engineer, with the aid of prepared tables, to estimate the size and number of lamps which will be required to produce the desired illumination. The determination of reflection factors with precision is one of the most difficult feats in photometry, and previous to this time, so far as the author knows, no method has been proposed which will give accurate results and which may be applied to the measurement of surfaces in place. All previous methods, with the exception of one, involve laboratory measurements, and the one exception does not give correct results.

\section{EARLIER REFLECTOMETERS}

If one considers the way in which the light flux is reflected, it is evident that in all cases except that of pure specular reflection any method of measuring reflection factors must inevitably involve an integration of the reflected flux. This practically limits the method to some application of the integrating sphere.

A search of the scientific literature reveals only a few articles on this subject. The information obtained shows that several methods have been proposed, but apparently nothing has been done as yet to standardize the method. In all except one of the methods of which descriptions were found the test surface is compared in some way with a standard surface, but little is said about the initial standardization of the reference standard surface. Even though the method of comparison may be faultless, the values obtained are merely relative to that of the standard surface, and if it is incorrectly evaluated they will evidently be subject to the same error. A number of the methods which have been used in the past have been seriously in error because of the assignment of an incorrect value to the standard surface used. A few of the methods used will be briefly described.

Apparently the first definite proposal of an instrument for the measurement of diffuse-reflection factors was made in I912 by Dr. Nutting. (See Bibliography, 2.) His instrument consists of a highly polished nickeled ring $150 \mathrm{~mm}$ in diameter and $32 \mathrm{~mm}$ high, through which projects the nose of a König-Martens polariza- 
tion photometer. An illuminated diffusing glass plate is placed on one side of the ring and the test surface on the other, and the photometer is arranged to view the two surfaces at an angle of about $75^{\circ}$ from the normal. He describes it thus: "The principle of the method is that of two parallel infinite planes, one of which is a diffuse illuminator and the other the surface whose reflecting power is to be determined. The relative brightness of the two planes is then the reflecting power of the nonluminous plane." Judging by the low value assigned to magnesium carbonate by this instrument, it apparently gives results which are very seriously in error. Some of the factors which may contribute to this error are as follows:

(a) The instrument is based on the principle of parallel infinite planes, whereas it employs planes of very limited area, bounded by a nickeled ring which is far from a perfect reflector.

(b) The distribution of illumination over the two planes is seriously disturbed by the presence of the nose piece of the polarization photometer and does not have the ideal distribution which is assumed.

(c) No surface obeys the cosine law of emission perfectly, and the brightness of the surface at a very oblique angle is usually very appreciably lower than that of a perfect diffuser emitting the same total flux.

A method devised and used by Mr. W. F. Little (see Bibliography, 3) at the Electrical Testing Laboratories consists in the projection of a beam of light through a small hole in the wall of an integrating sphere onto the test surface, placed near the center of the sphere. In this method the brightness of the observation window when the test surface is in place, compared with that when the standard surface is used, is substantially the same as the ratio of the reflection factors of the two surfaces. Evidently this method is limited by the accuracy of the value assigned to the standard surface, but a slight modification would make it an absolute method. If the light beam is first projected onto the sphere wall at a point unscreened from the observation window, and is next projected onto the test surface, screened from the window, the ratio of the brightness of the window in the second case to that in the first case is the numerical value of the reflection factor of the test surface. In this method the area of the test surface should be small with respect to the sphere surface. Another method of using the sphere would be to determine the reflection factor of its surface by a method which will presently be described, 
then to determine the relative values of test and sphere surface by projecting the beam first on one, then on the other, the illuminated spot being screened from the observation window in each case. The greatest practical difficulty in the application of any one of these three methods of using the sphere is the realization of a narrow beam of light which is of a sufficiently high intensity and at the same time is so concentrated that none of it is incident anywhere except on the test surface. The two modifications of Mr. Little's methods pointed out by the author require the sphere surface to be uniform in reflecting power.

In I916 Dr. Rosa (see Bibliography, 4) and the present author described and applied a method of measuring the reflection factor of the surface of an integrating sphere. In consists in the determination of the ratio of the average illumination received by the sphere surface from the test lamp to the total illumination of the sphere surface by both direct and reflected light, the numerical value of this ratio being the absorption factor of the sphere surface. The absorption factor of an 88-inch sphere at the Bureau of Standards, when the surface was fresh, was found to be 7.5 per cent. Since magnesium oxide and carbonate have long been considered, and probably are, the whitest substances in existence, this test set the lowest possible limit for their reflection factors, and definitely established the fact that the value of 88 per cent, given by Dr. Nutting's reflectometer; was considerably in error. This sphere method of determining the reflection factor of the sphere surface is by far the most precise method which it is possible to devise, but it is evidently very limited in its application.

In I9I 7 Mr. M. Luckiesh (see Bibliography, 5) described a new relative method for measuring reflection factors. An opal glass globe, such as is used in lighting fixtures, is mounted in a white box. The globe is surrounded by four or more lamps, symmetrically placed. The globe has an opening at the bottom, against which is placed the object to be tested. A brightness photometer views the test object, its brightness being compared with that of a standard surface of known reflection factor. The results of tests with this instrument may be in error because of the fact that the photometer views the test object at a fixed angle, and that the brightness at that angle may depend very largely on the amount of specular reflection of the object, but it is probable that this error would not be very large. The instrument will, however, give incorrect results if the standard surface is incorrectly evaluated. 


\section{THE NEW ABSOLUTE REFLECTOMETER}

In I916 the author worked out the complete theory of a reflectometer which was to be an absolute instrument, and shortly thereafter the experimental instrument used in these tests was constructed. The few tests which were made gave good results, but for lack of time very little more work was done with the instrument until within the past few months. No publication of the theory was made because insufficient work had been done to completely verify it, but it has now been verified by extensive experiments, as will be shown later.

At the convention of the Illuminating Engineering Society in Chicago, in October, 1919, in the discussion of two of the papers presented, the author called attention to the fact that the value of 88 per cent for magnesium carbonate was much too low, and also

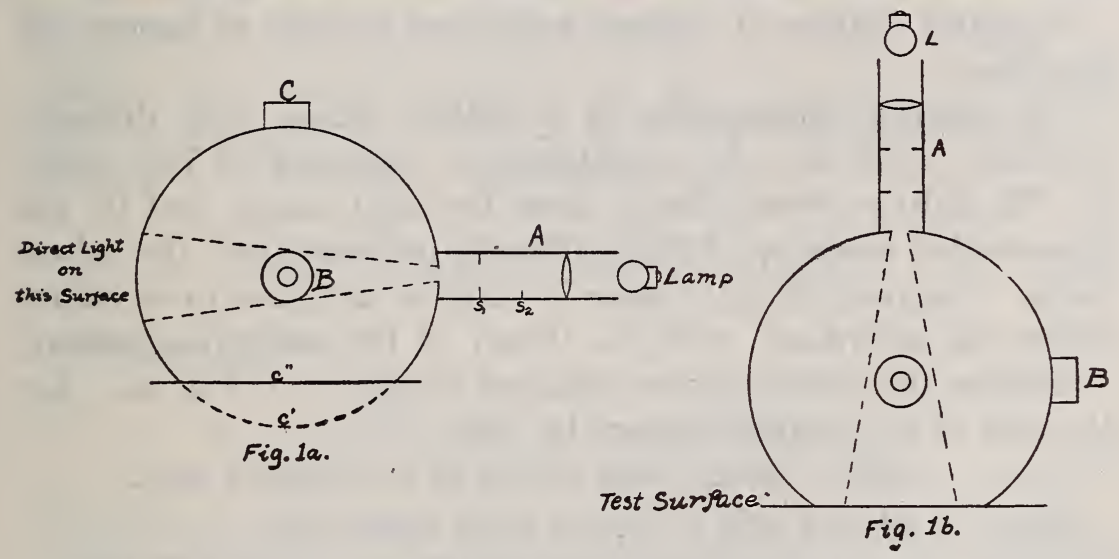

FIG. 1.-The new type "absolute" reflectometer

briefly described the instrument which it is the object of this paper to describe more fully. (See Bibliography, 6.) In a revision of his discussion, prepared shortly after the convention, the author stated that the reflection factor of magnesium carbonate was approximately 96 per cent, that being slightly lower than a value which he obtained by one measurement by point-by-point methods. ${ }^{2}$

The reflectometer consists of a small sphere arranged as shown in Fig. I. Light from a small lamp is projected through tube A

2 The absolute method for the determination of the reflection factor of magnesium carbonate which was described by another author in a recent publication is merely a modification of the method which the present author described in Chicago, and describes more fully here, and is strictly limited to the determination of the reflection factor of any surface which can be embodied in a hollow sphere. His method uses an incomplete sphere divisible into two fractional parts, whereas the author described the used of the complete sphere and one fractional part. 
onto the inner sphere wall. At $B$ is a small hole through which the opposite wall can be viewed by a brightness photometer. The segment of sphere surface $c^{\prime}$ is cut off by a plane, leaving the opening $c^{\prime \prime}$. The test surface is placed over this opening, and the direct light is projected onto the sphere wall or the test surface, depending on whether the lighting tube is placed at $A$ or $C$. The attachments are so constructed that their positions are interchangeable. In the experimental instrument constructed and used in the tests the area $c^{\prime}$, the portion cut off, was Io per cent of the total sphere area.

\section{THEORY OF REFLECTOMETER}

If a plane surface is illuminated by an infinite plane of uniform brightness $b$ (candles per unit area), the flux received by unit area of the illuminated plane is $\pi b$ lumens. (See Bibliography, 7.)

A perfect diffuser of uniform brightness $b$ emits $\pi b$ lumens per unit area.

The interior illumination of a hollow sphere with diffusely reflecting walls may be considered as composed of two parts: (a) The light received directly from the light source, and (b) the illumination made up of light diffusely reflected from the sphere walls. The part (b) is the same in value at all points in an empty sphere, in accordance with the theory of the integrating sphere.

Assume the hollow sphere arranged as shown in Fig. I $a$. Let the area of the complete sphere be unity.

Let $c^{\prime}=$ ratio of sphere area cut off to total sphere area.

Let $c^{\prime \prime}=$ ratio of area of hole to total sphere area.

Let $a=\mathrm{I}-c^{\prime}=$ fraction of total sphere area which remains.

Let $m=$ diffuse reflection factor of sphere surface.

Let $m_{x}=$ diffuse reflection factor of test surface.

Let $F=$ total light flux (lumens) received from lamp.

Let $b_{\mathrm{o}}=$ average brightness (candles per unit area) of sphere wall due to reflected light only when hole is uncovered.

Let $b_{\mathrm{x}}=$ brightness when hole is covered with a flat test surface having a diffuse reflection factor $m_{\mathbf{x}}$.

Let $b=$ brightness when test surface has reflection factor $m$.

When the hole is uncovered, the escaping light flux may be considered as composed of two parts. The portion of first reflected flux which escapes through the hole is $c^{\prime} m F$, since it is the once reflected flux which would ordinarily be received by the portion of the surface which has been removed. The second part is the 
flux due to the average brightness $b_{0}$ of the sphere walls, winich in turn is due to reflected light only. Its effect in the plane $c^{\prime \prime}$ is equivalent to that of an infinite luminous plane of brightness $b_{0}$. Hence, this second part is $\pi b_{0} c^{\prime \prime}$, and the total flux escaping through the hole is

$$
F^{\prime}=c^{\prime} m F+\pi b_{0} c^{\prime \prime} .
$$

The total flux received by the remaining portion of the sphere surface is

$$
\frac{\pi b_{o} a}{m}+F
$$

the amount absorbed being

$$
(\mathrm{I}-m)\left[\frac{\pi b_{0} a}{m}+F\right]
$$

Neglecting the small amount of light flux lost through the lighting and observing windows, the sum of $(I)$ and (2) must be equal to the total flux received, since it all escapes through the opening or is absorbed. Therefore we have

$$
\pi b_{0} c^{\prime \prime}+c^{\prime} m F+(\mathrm{I}-m)\left[\frac{\pi b_{0} a}{m}+F\right]=F .
$$

Solving (3) for $b_{0}$,

$$
b_{0}=\frac{m^{2} F\left(\mathrm{I}-c^{\prime}\right)}{\pi\left[m c^{\prime \prime}+a(\mathrm{I}-m)\right]}=\frac{m^{2} F a}{\pi\left[m c^{\prime \prime}+a(\mathrm{I}-m)\right]} .
$$

If the hole is covered with a flat surface having a reflection factor $m_{\mathrm{x}}$, then $\left(\mathrm{I}-m_{\mathrm{x}}\right)$ times the flux incident on the test surface will be absorbed. In that case we find that

$$
\left(\mathrm{I}-m_{\mathrm{x}}\right)\left[\pi b_{\mathbf{x}} c^{\prime \prime}+c^{\prime} m F\right]+(\mathrm{I}-m)\left[\frac{\pi b_{\mathbf{x}} a}{m}+F\right]=F .
$$

Solving for $b_{\mathrm{x}}$ we have

$$
b_{\mathrm{x}}=\frac{m^{2} F\left(a+c^{\prime} m_{\mathrm{x}}\right)}{\pi\left[c^{\prime \prime} m\left(\mathrm{I}-m_{\mathrm{x}}\right)+a(\mathrm{I}-m)\right]}
$$

If $m_{\mathrm{x}}=m$-that is, if the test surface has the same reflection factor as the sphere surface-then (6) becomes

$182802^{\circ}-20-2$

$$
b=\frac{m^{2} F\left(a+c^{\prime} m\right)}{\pi(\mathrm{I}-m)\left(c^{\prime \prime} m+a\right)} .
$$


It is possible to measure the relative brightness of the sphere wall under the various conditions, and from these $m$ and $m_{\mathrm{x}}$ can be computed.

Let

$$
\frac{b}{b_{o}}=K \text { and } \frac{b_{x}}{b_{o}}=K_{x}
$$

Then

$$
K=\frac{\frac{m^{2} F\left(a+c^{\prime} m\right)}{\pi(\mathrm{I}-m)\left(c^{\prime \prime} m+a\right.}}{\frac{m^{2} F a}{\pi\left[m c^{\prime \prime}+a(\mathrm{I}-m)\right]}}=\frac{\left(m c^{\prime}+a\right)\left[m c^{\prime \prime}+a(\mathrm{I}-m)\right]}{a\left(m c^{\prime \prime}+a\right)(\mathrm{I}-m)} .
$$

Clearing of fractions and collecting,

$m^{2}\left[c^{\prime}\left(c^{\prime \prime}-a\right)+K a c^{\prime \prime}\right]+m a\left[c^{\prime}+\left(c^{\prime \prime}-a\right)(\mathrm{I}-K)\right]+a^{2}(\mathrm{I}-K)=\mathrm{O}$.

Since $c^{\prime}, c^{\prime \prime}$, and $a$ are dimensional constants of the sphere and $K$ can be measured, $m$, the reflection factor of the sphere surface, can be evaluated from this equation.

$$
\begin{gathered}
K_{\mathbf{x}}=\frac{\frac{m^{2} F\left(a+c^{\prime} m_{\mathbf{x}}\right)}{\pi\left[c^{\prime \prime} m\left(\mathrm{I}-m_{\mathbf{x}}\right)+a(\mathrm{I}-m)\right]}}{\frac{m^{2} F a}{\pi\left(m c^{\prime \prime}+a(\mathrm{I}-m)\right]} \cdot}=\frac{\left(a+c^{\prime} m_{\mathbf{x}}\right)\left[m c^{\prime \prime}+a(\mathrm{I}-m)\right]}{a\left[m c^{\prime \prime}\left(\mathrm{I}-m_{\mathbf{x}}\right)+a(\mathrm{I}-m)\right]} \\
m_{\mathbf{x}}=\frac{a\left(K_{\mathbf{x}}-\mathrm{I}\right)\left[c^{\prime \prime} m+a(\mathrm{I}-m)\right]}{a c^{\prime}(\mathrm{I}-m)+m c^{\prime \prime}\left(c^{\prime}+K_{\mathbf{x}} a\right)}
\end{gathered}
$$

The above equations have been derived for the case where the direct light from the lamp is incident on the sphere wall. While it is possible to use the reflectometer with that arrangement, it is much more sensitive to changes of reflection factor of the test surface, and therefore more accurate, if it is arranged so that the direct light is incident on the test surface. The most convenient method is to use the former arrangement and determine the value of $m$ from equation (9), then arrange the instrument so that the light is incident directly on the test surface, as shown in Fig. $\mathrm{I} b$. By using for a reference standard a surface painted with the sphere paint it is then possible to evaluate in absolute measure the reflection factor of any test surface. In practical work, however, it is preferable to use a less perishable reference standard. A rough-surface milk glass makes a satisfactory reference standard, as it is easily restored by washing. It can be standardized by 
means of observations and use of equations (I I) and (I 7 ). The theory of the instrument when so used is as follows:

Let $c^{\prime}, c^{\prime \prime}, a, m, m_{\mathbf{x}}$, and $F$ have the same meaning as before.

Let $b^{\prime}=$ brightness of sphere surface when the hole is covered with a test surface of factor $m_{\mathbf{x}}$.

Let $R=\frac{\text { value of } b^{\prime} \text { when hole is covered with surface } m^{\prime \prime}}{\text { value of } b^{\prime} \text { when hole is covered with surface } m^{\prime}}$

Since unit area of the sphere surface of brightness $b^{\prime}$ emits $\pi b^{\prime}$ lumens, it must be receiving $\frac{\pi b^{\prime}}{m}$ lumens. Hence the sphere area $a$ absorbs $(\mathrm{I}-m) \frac{\pi b^{\prime} a}{m}$ lumens. The test surface receives $F+c^{\prime \prime} \pi b^{\prime}$ and absorbs $\left(\mathrm{I}-m_{\mathrm{x}}\right)\left(F+c^{\prime \prime} \pi b^{\prime}\right)$ lumens. Hence, since all the light is absorbed except that negligible amount which escapes through the lighting and observing holes, we have

$$
(\mathrm{I}-m) \frac{\pi b^{\prime} a}{m}+\left(\mathrm{I}-m_{\mathbf{x}}\right)\left(\dot{F}+c^{\prime \prime} \pi b^{\prime}\right)=F .
$$

Solving for $b^{\prime}$,

$$
b^{\prime}=\frac{m m_{\mathbf{x}} F}{\pi\left[a(\mathrm{I}-m)+c^{\prime \prime} m\left(\mathrm{I}-m_{\mathbf{x}}\right)\right.},
$$

$$
R=\frac{\frac{m m^{\prime \prime} F}{\pi\left[a(\mathrm{I}-m)+c^{\prime \prime} m\left(\mathrm{I}-m^{\prime \prime}\right)\right]}}{\frac{m m^{\prime} F}{\pi\left[a(\mathrm{I}-m)+c^{\prime \prime} m\left(\mathrm{I}-m^{\prime}\right)\right]}}=\frac{m^{\prime \prime}\left[a(\mathrm{I}-m)+c^{\prime \prime} m\left(\mathrm{I}-m^{\prime}\right)\right]}{m^{\prime}\left[a(\mathrm{I}-m)+c^{\prime \prime} m\left(\mathrm{I}-m^{\prime \prime}\right)\right]}
$$

Solving for $m^{\prime \prime}$,

$$
m^{\prime \prime}=\frac{R m^{\prime}\left[a+m\left(c^{\prime \prime}-a\right)\right]}{a(\mathrm{I}-m)+c^{\prime \prime} m\left(\mathrm{I}-m^{\prime}+R m^{\prime}\right)}
$$

In the special case where $m^{\prime}=m$, we have

$$
m^{\prime \prime}=m_{\mathbf{x}}=\frac{R m\left[a(\mathrm{I}-m)+m c^{\prime \prime}\right]}{\left[(\mathrm{I}-m)\left(a+c^{\prime \prime} m\right)+R m^{2} c^{\prime \prime}\right]} .
$$

\section{EXPERIMENTAL RESULTS}

In order to verify the theory of this instrument a graded series of test objects was made up. Neutral gray objects were obtained by mixing black drawing ink and lampblack with a white cement (Keene's Fine). They were surfaced with coarse sandpaper, resulting in fairly good diffusers. Those having reflection factors below 50 per cent were better diffusers than those above that value. 
This appears to be characteristic of this material and possibly of others, and may be treated more fully in a future paper. The gray objects, after having been made up for about four or five months, were found to have faded somewhat, and hence are not satisfactory for permanent reflection factor standards.

The test objects were next tested for reflection factors by means of the apparatus shown in Fig. 2. The objects were illuminated normally by a 60 -watt vacuum tungsten lamp, and the surface brightness was measured by point-by-point observations. The amount of flux reflected was then calculated by applying the proper factors, and since the incident flux was known, the ratio

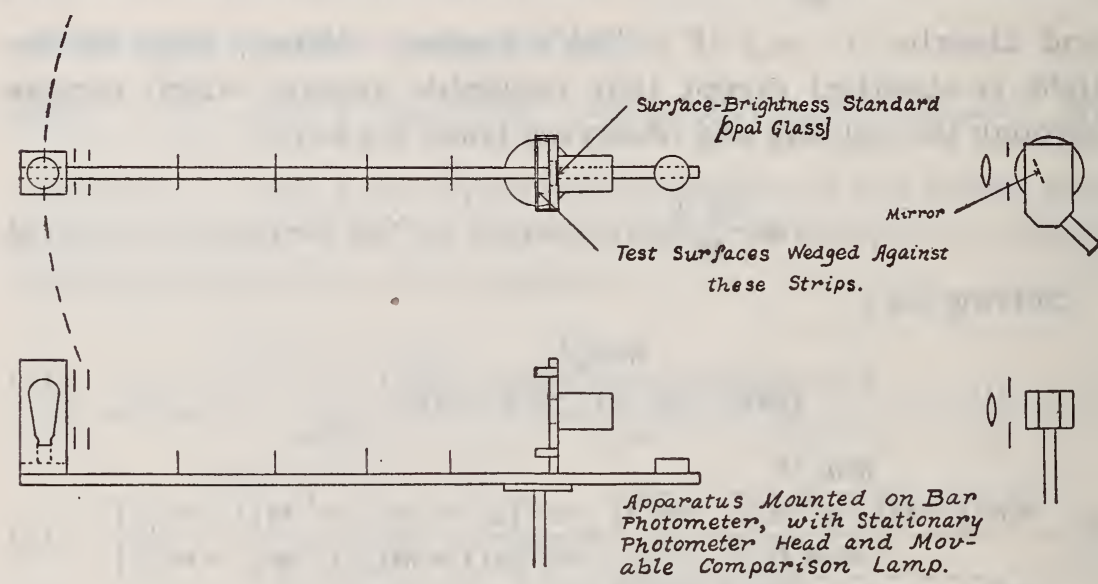

Fig. 2.-Apparatus used in determining reflection factors by measurements of surface brightness at $10^{\circ}$ intervals

of the reflected to the incident flux gave the reflection factors of the test surfaces. The greatest difficulty in that method of measurement is the standardization of the apparatus to read surface brightness, as it involves very considerable practical difficulties, chief among which is the great intensity step which must be taken. Much work was done on this part of the problem, and it is believed that the uncertainty in that standardization was not very large.

A number of the cement standards and a block of magnesium carbónate were measured by both methods of using the instrument as described above. By the use of equation (9) the reflection factor of the sphere surface was found to be 88.3 per cent. This value was then substituted in the equation and the reflection factors of the various test objects were computed. The results of the measurements in the three different ways are shown in Table I. 
TABLE 1.-Reflection Factors by Three Methods of Measurement

Test object
$\ldots$

It will be noted that the agreement between columns 2 and 3 is almost perfect, and that for reflection factors above 80 per cent the fourth column is in satisfactory agreement. For factors below 80 per cent the method represented by the fourth column is rather insensitive, the error in the result being larger than the error of the photometer reading with the actual conditions existing in the experimental instrument. This is clearly shown by the curves in Fig. 3. When the direct light is incident on the test surface the error in the factor is always less than the error of the reading. Hence it may safely be stated that at least a part of the discrepancy between columns 2 and 4 may be attributed to experimental error. The fact that the differences are all in the same direction for the objects having low reflection factors might lead to the belief that not all the discrepancy could be explained this way, and that it might be necessary to look farther for an additional reason. It is quite probable that the reflection factor actually is greater when an object is diffusely illuminated than when the light is incident normally, since some of the incident rays strike the surface at very oblique angles and do not penetrate the surface. If this explanation is correct, it naturally follows that the effect would be greater the lower the reflection factor of the surface. The sensitivity of the instrument when used with diffused illumination of the test surfaces would be greatly increased if the reflectometer were painted with a paint having a higher reflecting power, and it might then be possible to determine whether this is 
a real departure from the theory as developed. The author has made up paints having a factor of about 94 per cent, which would be much better for this application.

It will be noted that the measurements by the three methods give about 99 per cent for magnesium carbonate, whereas the previously accepted value was only 88 per cent It did not seem possible that its value could be so high, and the author was reluctant to accept that value as reliable in spite of the agreement of the three methods, unless verified by still another method. Hence steps were taken to verify it by a fourth absolute method,

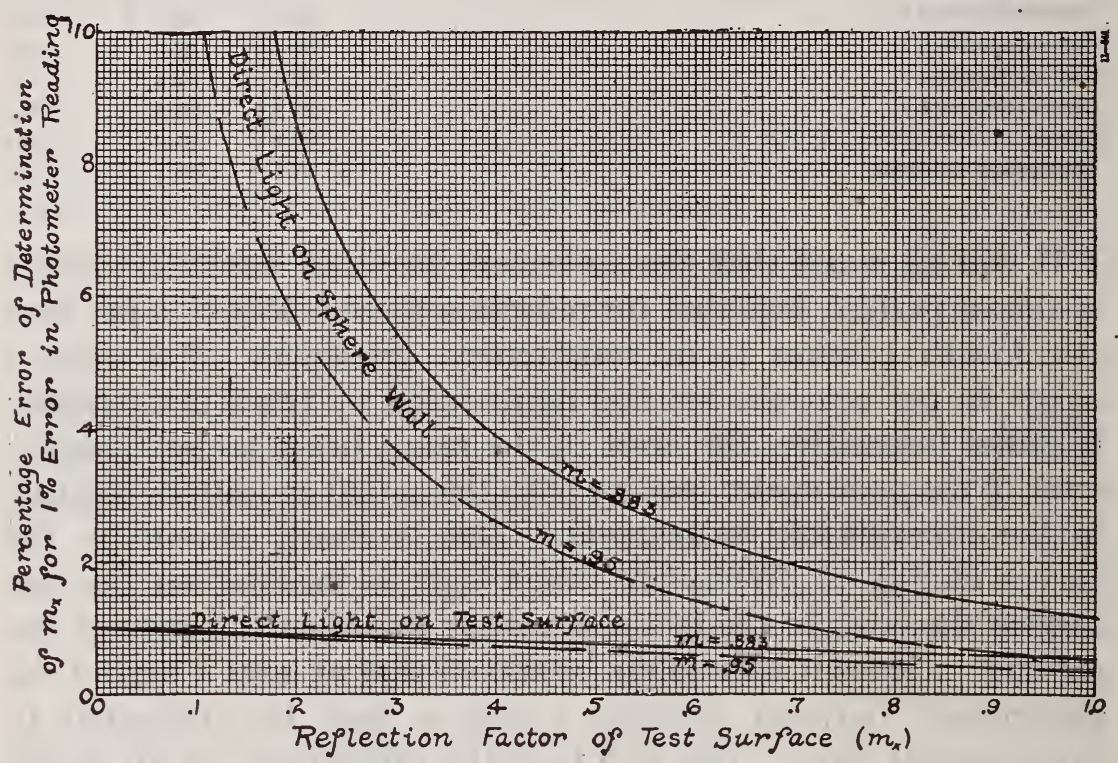

FIG. 3.-Curves showing how the precision of determinations with the new reflectometer varies with different conditions

which was described above as a modification of the sphere method used by Mr. Little. For this purpose a small disk of magnesium carbonate wås made up from the block previously tested. It was placed in a sphere and a very narrow beam of light was projected through a small hole in the sphere wall onto the sphere surface at a point unscreened from the observation window, then onto the magnesium-carbonate disk so placed that none of the first reflected light from it could reach the observation window. The ratio of the brightness of the window in the second case to that in the first case is the reflection factor of the test surface. This measurement gave a factor of 98.9 per cent which, on the basis of the consistency of the photometric readings, appeared to be thoroughly reliable. 
Hence it appears that the reflection factor of this particular block of magnesium carbonate is 99 per cent. Another block, obtained about three weeks later from the same source, has a factor of approximately 98 per cent while another block which has been in the laboratory for about two or three years is appreciably darker than either of these. No investigation has bəen made of the reproducibility of such surfaces for precision standards.

It should be pointed out that magnesium carbonate is not a perfect diffuser, hence this must be taken into account if it is desired to use it as a standard of surface brightness. The block which was tested by point-by-point measurements was surfaced by scraping with a sharp glass edge. When illuminated normally its brightness at about $50^{\circ}$ from the normal to the surface was the same as that of a theoretically perfect diffuser emitting the same total flux.

\section{EFFECT OF SPECULAR REFLECTION FROM TEST SURFACES}

As mentioned above, the surfaces with which these tests were carried out were fairly good diffusers. Some other surfaces which have been examined by point-by-point measurements have been ordinary semimat surfaces. An examination of these data shows that when the light is incident normally the angle at which the surface has the same brightness as a perfect diffuser emitting the same total flux is usually about $50^{\circ}$, which is also approximately the angle between the normal and the line joining the observation window and the center of the test surface. The difference between the surface brightness of the test surface at $50^{\circ}$ and a perfect diffuser emitting the same total flux rarely exceeds 3 to 5 per cent and is usually below 3 per cent. The effect of a deviation of 5 per cent from perfect diffusion at $50^{\circ}$ for a surface of reflection factor 80 per cent has been calculated for a reflectometer having Io per cent of its area cut off, and painted with a paint having a reflection factor of 90 per cent. The calculation shows that the error of the determination would be less than 0.5 per cent. Hence it appears safe to state that the error of determination due to specular reflection will not exceed 2 per cent in a reflectometer having these dimensions, unless an excessive amount of the specular reflection of direct light is incident on the observation window. This will not hold true unless the specular reflection takes place only at the first surface. 
If it is desired to determine the reflection factor of a mirror, this may be done by first directing a narrow beam of light into the opening of the reflectometer, then let the same beam be reflected from the mirror and be directed into the reflectometer when the mirror is placed at an appreciable distance from the reflectometer. The ratio of the brightness of the observation window in the second case to that in the first will be the reflection factor of the mirror.

\section{PRECAUTIONS IN USE OF REFLECTOMETER}

In the use of the reflectometer as described above certain precautions are necessary. Some of them are as follows:

(a) The dimensions should be precisely determined in order to fix the values of the constants $c^{\prime}, c^{\prime \prime}$, and $a$.

(b) In painting the sphere and the flat surface by means of which its reflection factor is determined, care must be taken to make the flat surface as nearly as possible the same in reflecting power as the sphere surface.

(c) When calculating the reflection factor of the sphere surface by the use of the equation (9), the figures should be carried out as far as possible, as it involves differences of numbers which are nearly equal, and a small error in calculation may make an appreciable error in the result. The use of logarithms for this step is recommended, though slide-rule calculations are sufficiently accurate in working up test data for observations taken with direct light on the test surfaces.

(d) When the direct light is incident on the sphere wall, care must be taken to prevent any direct light from escaping through the large opening $c^{\prime \prime}$.

(e) Few, if any, paints will remain absolutely constant in reflecting power, hence the reflection factor of the sphere surface, when once determined, should not be assumed constant thereafter but should be checked frequently. This can be done by means of test objects standardized when the paint in the sphere is fresh. Depolished milk glass is excellent for this purpose, and white blotting paper would probably be satisfactory, too. . If a sufficient number of such objects, covering a wide range of reflection factors, were standardized very carefully when the surface was fresh, they could be used to establish an empirical calibration of the reflectometer at any time, without the necessity of solving the mathematical equations. Two standards having reflection factors of about 90 and $5^{\circ}$ per cent could be used to determine the reflection 
factor of the reflectometer surface at any time by the use of equation (I5), and the calibration curve could then be calculated and plotted for ready reference. An oil paint is not satisfactory for painting the reflectometer, on account of its change with time. If the paint used is fairly constant, the method first described in the theory of the reflectometer for determining its reflection factor would be entirely satisfactory, but with any paint which changes very much there is the danger that the reflectometer surface and the flat surface painted with the same paint would not change at the same rate.

The most symmetrical arrangement of the reflectometer is obtained if the observation hole and side lighting hole are about $90^{\circ}$ from the center of the portion cut off, and $90^{\circ}$ from each other. This is the arrangement shown in Fig. I $a$.

\section{CONCLUSION}

In conclusion the most important points brought out by this paper may be summarized as follows:

I. Five "absolute" methods of measuring reflection factors are described, at least three of which are apparently new. Measurements on magnesium carbonate by four of these methods give values which are in excellent agreement.

2. The numerical value of the reflection factor for manesium carbonate which has been in use for many years-viz, 88 per cent-is in error. This fact was first publicly pointed out by the author at Chicago in October, I9r9. The actual value of its diffuse reflection factor is approximately 99 per cent, but the degree of reproducibility of this value with materials from different sources is unknown.

3. The method described above for the use of an incomplete sphere as a reflectometer furnishes two new absolute methods for the determination of diffuse reflection factors. The determination of the reflection factor of the sphere surface is only an incidental step in the use of the instrument, and is not its principal object.

4. The instrument just described should find a large field of usefulness in photometry and illuminating engineering, and furnishes a method of measuring the reflection factors of surfaces in situ. Apparently no other instrument has been proposed for this purpose which is accurate and portable. It can be adapted for use with any good type of portable photometer. 
5. It should be strongly emphasized that the reflection factor of any colored surface is dependent on the color of the incident light and that measurements by this or any other. method will give its reflection factor only under the particular conditions of the test. Hence under such conditions the principal value of the measurement is to indicate the approximate value of the factor, but this is all that is usually required.

\section{BIBLIOGRAPHY}

(I) A. P. Trotter, Diffused Reflection and Transmission of Light, Illuminating Engineer, London, pp. 243-267; September, Igrg.

(2) P. G. Nutting, A New Method and Instrument for Determining the Reflecting Power of Opaque Bodies, Trans. Illum. Eng. Soc., 7, p. 412; 1912.

(3) Henry A. Gardner, The Light-Reflecting Values of White and Colored Paints, Jour. Franklin Institute, 181, p. 99; 1916.

(4) E. B. Rosa and A. H. Taylor, The Integrating Photometric Sphere; its Construction and Use, Trans. Illum. Eng. Soc., 11, p. 453; I916.

5) M. Luckiesh, Measurement of Reflection Factor, Elec. World, 69, p. 958; 1917. Journal of Optical Society of America; January-March, I9r9.

(6) Discussion by A. H. Taylor, Trans. Illum. Eng. Soc., 15, p, I32, March 20, 1920.

(7) E. B. Rosa, Photometric Units and Nomenclature, B. S. Bulletin, 6, p. 543; I909-IO. Also B. S. Sci. Paper No. I4I.

WASHINGTON, March II, I92O. 